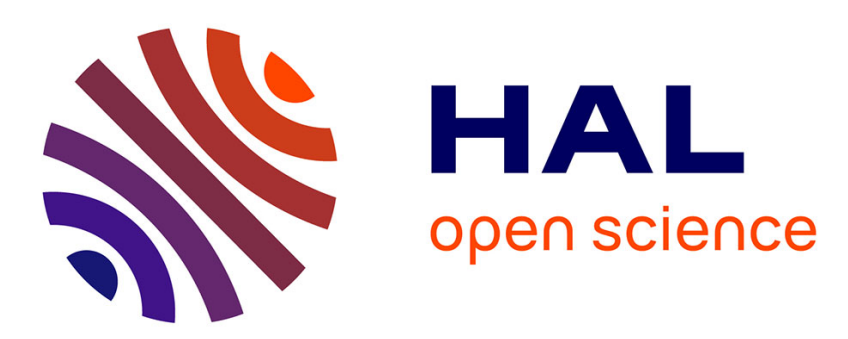

\title{
Efficient dominant point detection based on discrete curve structure
}

Phuc Ngo, Hayat Nasser, Isabelle Debled-Rennesson

\section{To cite this version:}

Phuc Ngo, Hayat Nasser, Isabelle Debled-Rennesson. Efficient dominant point detection based on discrete curve structure. IWCIA 2015, Nov 2015, Kolkata, India. pp.143-156, 10.1007/978-3-31926145-4_11. hal-01218285

\section{HAL Id: hal-01218285 \\ https://inria.hal.science/hal-01218285}

Submitted on 20 Oct 2015

HAL is a multi-disciplinary open access archive for the deposit and dissemination of scientific research documents, whether they are published or not. The documents may come from teaching and research institutions in France or abroad, or from public or private research centers.
L'archive ouverte pluridisciplinaire HAL, est destinée au dépôt et à la diffusion de documents scientifiques de niveau recherche, publiés ou non, émanant des établissements d'enseignement et de recherche français ou étrangers, des laboratoires publics ou privés. 


\title{
Efficient dominant point detection based on discrete curve structure
}

\author{
Phuc Ngo ${ }^{1,2}$, Hayat Nasser ${ }^{1,2}$, Isabelle Debled-Rennesson ${ }^{1,2}$ \\ ${ }^{1}$ Université de Lorraine, LORIA, UMR 7503, Vandoeuvre-lès-Nancy, F-54506, France \\ ${ }^{2}$ CNRS, LORIA, UMR 7503, Vandoeuvre-lès-Nancy, F-54506, France \\ \{hoai-diem-phuc.ngo, hayat.nasser, isabelle.debled-rennesson\}@loria.fr
}

\begin{abstract}
In this paper, we investigate the problem of dominant point detection on digital curves which consists in finding points with local maximum curvature. Thanks to previous studies of the decomposition of curves into sequence of discrete structures [5-7], namely maximal blurred segments of width $\nu$ [13], an initial algorithm has been proposed in [14] to detect dominant points. However, an heuristic strategy is used to identify the dominant points. We now propose a modified algorithm without heuristics but a simple measure of angle. In addition, an application of polygonal simplification is as well proposed to reduce the number of detected dominant points by associating a weight to each of them. The experimental results demonstrate the efficiency and robustness of the proposed method.
\end{abstract}

Keywords: Dominant point, polygonal simplification, discrete structure

\section{Introduction}

Dominant points of discrete curves are identified by Attneave [2] as the local maximum curvature points on a curve. Such points content a rich information which is sufficient to characterize this curve. They play a critical role in curve approximation, image matching and in other domains of computer vision. Many works have been conducted regarding the dominant point detection $[1,3,4,8-12$, $14,17-20]$ and surveys are presented in $[1,12,14]$. Several problems have been identified in the different approaches: time computation, number of parameters, selection of starting point, bad results with noisy curves, ...

Nguyen et. al proposed in [14] a new sequential method issued from theoretical results of discrete geometry, it only requires to set one parameter, it is invariant to the choice of the starting point and it naturally works with general curves: possibly being noisy or disconnected. It relies on the geometrical structure of the studied curve, in particular the decomposition of the curve into maximal blurred segments for a given width $[13,6]$. However at the end of the proposed method, the choice of dominant points is done with an heuristic strategy.

In this paper, an improvement of this dominant point detection algorithm is proposed with an efficient choice of dominant points by considering a simple 
measure of angle. Furthermore, an algorithm of polygonal simplification is also proposed to reduce the number of detected dominant points while preserving the principal angular deviations in the discrete curve of the shape border.

The paper is organized as follows: in section 2 , we recall results of discrete geometry used in this paper to analyze a curve. Then, in section 3 , we describe the previous dominant point detection algorithm [14] and propose improvements. In section 4, we present experimental results, comparisons with previous methods and an application to polygonal simplification.

\section{Decomposition of a curve into maximal blurred segments}

We recall hereafter several notions concerning discrete lines [15], blurred segments [5] and maximal blurred segments [13] which are used in the paper.

Definition 1. A discrete line $\mathcal{D}(a, b, \mu, \omega)$, with a main vector $(b, a)$, a lower bound $\mu$ and an arithmetic thickness $\omega$ (with $a, b, \mu$ and $\omega$ being integer such that $\operatorname{gcd}(a, b)=1)$ is the set of integer points $(x, y)$ verifying $\mu \leq a x-b y<\mu+\omega$. Such a line is denoted by $\mathcal{D}(a, b, \mu, \omega)$.

Let us consider $\mathcal{S}_{f}$ as a sequence of integer points.

Definition 2. A discrete line $\mathcal{D}(a, b, \mu, \omega)$ is said to be bounding for $\mathcal{S}_{f}$ if all points of $\mathcal{S}_{f}$ belong to $\mathcal{D}$.

Definition 3. A bounding discrete line $\mathcal{D}(a, b, \mu, \omega)$ of $\mathcal{S}_{f}$ is said to be optimal if the value $\frac{\omega-1}{\max (|a|,|b|)}$ is minimal, i.e. if its vertical (or horizontal) distance is equal to the vertical (or horizontal) thickness of the convex hull of $\mathcal{S}_{f}$.

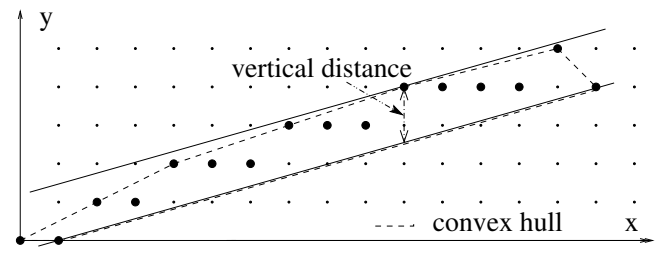

Fig. 1. $\mathcal{D}(2,7,-8,11)$, the optimal bounding line of the set of points (vertical distance $\left.=\frac{10}{7}=1.42\right)$.

This definition is illustrated in Fig. 1 and leads to the definition of the blurred segments.

Definition 4. A set $\mathcal{S}_{f}$ belongs to a blurred segment of width $\nu$ if its optimal bounding line has a vertical or horizontal distance less than or equal to $\nu$ i.e. if $\frac{\omega-1}{\max (|a|,|b|)} \leq \nu$. 
The notion of maximal blurred segment was introduced in [13]. Let $C$ be a discrete curve and $C_{i, j}$ a sequence of points of $C$ indexed from i to $\mathrm{j}$. Let us suppose that the predicate " $C_{i, j}$ is a blurred segment of width $\nu$ " is denoted by $B S(i, j, \nu)$.

Definition 5. $C_{i, j}$ is called a maximal blurred segment of width $\nu$ and noted $M B S(i, j, \nu)$ iff $B S(i, j, \nu), \neg B S(i, j+1, \nu)$ and $\neg B S(i-1, j, \nu)$.

An incremental algorithm was proposed in [13] to determine the sequence of all maximal blurred segments of width $\nu$ of a discrete curve $C$. The main idea is to maintain a blurred segment when a point is added (or removed) to (from) it. The two following important properties were proved.

Property 1 Let $M B S_{\nu}(C)$ be the sequence of width $\nu$ maximal blurred segments of the curve $C$. Then, $M B S_{\nu}(C)=\left\{M B S\left(B_{0}, E_{0}, \nu\right), M B S\left(B_{1}, E_{1}, \nu\right), \ldots, M B S\left(B_{m-1}, E_{m-1}, \nu\right)\right\}$ and satisfies $B_{0}<B_{1}<\ldots<B_{m-1}$. So we have: $E_{0}<E_{1}<\ldots<E_{m-1}$ (see Fig. 2).

Property 2 Let $L(k), R(k)$ be the functions which respectively return the indexes of the left and right extremities of the maximal blurred segments on the left and right sides of the point $C_{k}$ in a discrete curve $C$. So, for each index $k$ such that $E_{i-1}<k \leq E_{i}$, we have $L(k)=B_{i}$, and, for each $k$ such that $B_{i} \leq k<B_{i+1}$, we have $R(k)=E_{i}$.

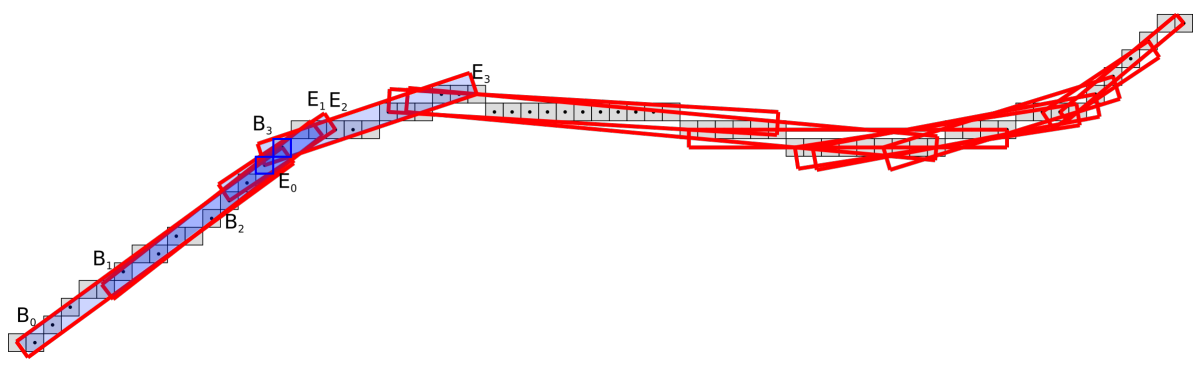

Fig. 2. A sequence of width 1.4 maximal blurred segments of the curve with grey pixels (each red box bounds the pixels of a maximal blurred segment of width 1.4). The indexes of the begin $\left(B_{i}\right)$ and end $\left(E_{i}\right)$ points of the four first maximal blurred segments are indicated $\left(B_{0}=0\right.$ and $E_{0}=15, B_{1}=5$ and $\left.E_{1}=17, \ldots\right)$. The points with blue frame are points in the common zone - intersecting zone - defined by these four first maximal blurred segments.

For a given width $\nu$, the sequence of the maximal blurred segments of a curve $C$ entirely determines the structure of $C$. It can be considered as an extension to thick curves of the notion of tangential cover introduced by Feschet and 
Tougne in [7] and used to obtain curvature profile or fast polygon approximation [8]. In [14], a method for dominant point detection, constructed from the sequence of maximal blurred segments, was given. In the next section, we propose an improvement of this method which implies new applications.

\section{Dominant point detection}

The sequence of maximal blurred segments of a curve provides important information about the geometrical structure of the studied curve. The width of the maximal blurred segments permits to work in different scales and to consider the noise eventually present in a curve $C$.

In [2], Attneave defined a dominant point (corner point) on a curve as a point of local maximum curvature. In this way, the method proposed in [14] uses a notion of region of support (ROS) of a point in a curve.

Definition 6. Width $\nu$ maximal left and right blurred segments of a point constitute its region of support (ROS). The angle between them is called the ROS angle of this point.

The method first chooses a width $\nu$ and computes $M B S_{\nu}(C)$, the sequence of maximal blurred segments of $C$. The main idea of the method is that dominant points of $C$ are localised in the common zones of successive maximal blurred segments of $C$ (see Fig. 2). Hereafter, we recall the properties and the heuristic strategy used in [14]. We show that problems can occur and propose a solution.

\subsection{Heuristic strategy of method [14]}

Let us now consider the common zone of more than 2 successive maximal blurred segments.

Proposition 1. The smallest common zone of successive width $\nu$ maximal blurred segments, whose slopes are either increasing or decreasing, contains a candidate as dominant point.

Property 3 A maximal blurred segment contains at most 2 dominant points.

Based on the previous propositions and properties, the algorithm proposed by Nguyen et al. in [14] consists in finding the smallest common zone induced by successive maximal burred segments of width $\nu$ which have either increasing or decreasing slopes. It is stated that such a zone contains the candidates of dominant point, since the points in this zone have the smallest ROS angle. By an heuristic strategy, the dominant point is identified as the middle point of this zone.

This heuristic is very effective, but sometimes leads to a non optimal solution for the polygonal simplification problem, as shown in the next section. 


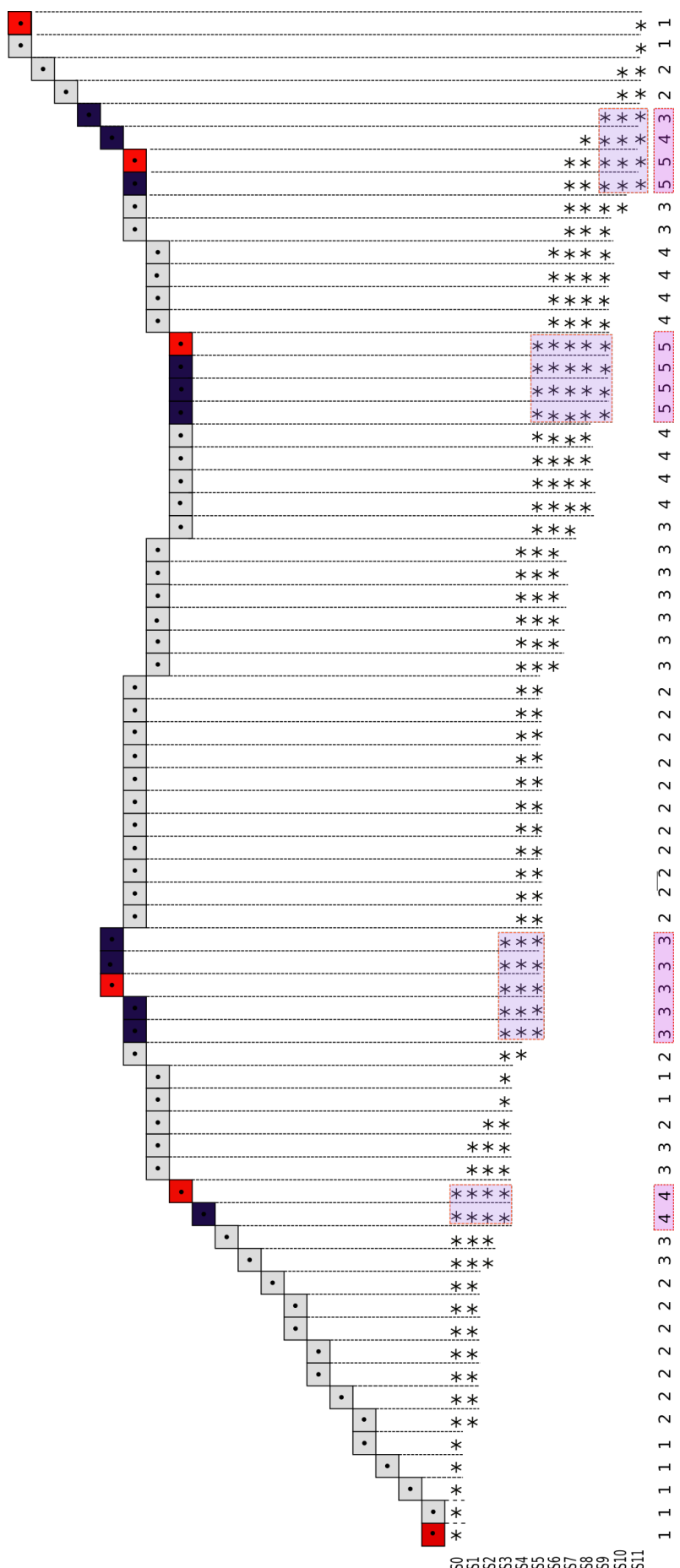

Fig. 3. Interest regions (common zones) obtained with the sequence of width 1.4 maximal blurred segments of the curve with grey pixels. 


\subsection{New dominant point detection algorithm}

In this section, we present a modified algorithm of [14] for detecting the dominant points of discrete curves with high accuracy.

Since the purpose of dominant point detection is to detect significant points with curvature extreme, we propose a new decision-making strategy of dominant point in common zones. For this, we use a simple measure, as a pseudo-curvature, estimated for each point located in the interest regions. In particular, we consider the $k$-cosine measure [16] adapted to the decomposition into width $\nu$ blurred segments of the input curve. More precisely, it is the angle between the considered point and the two left and right extremities of the left and right maximal blurred segments, involved in the localisation of the studied common zone and passing through the point. Obviously, the smaller angle, the greater curvature and thus the higher dominant character of the point. Accordingly, the dominant point is identified as the point, in the common zone, having a local minimum measure of angle.

The modified algorithm is given in Algorithm 1. It is similar to the one in [14] except for the selection of dominant points as described above. Moreover we simplify the process because we don't need to decompose the slopes of the maximal blurred segments located in the common zones into monotone sequences. Indeed, the angle computation permits to well localize the dominant point in each common zone.

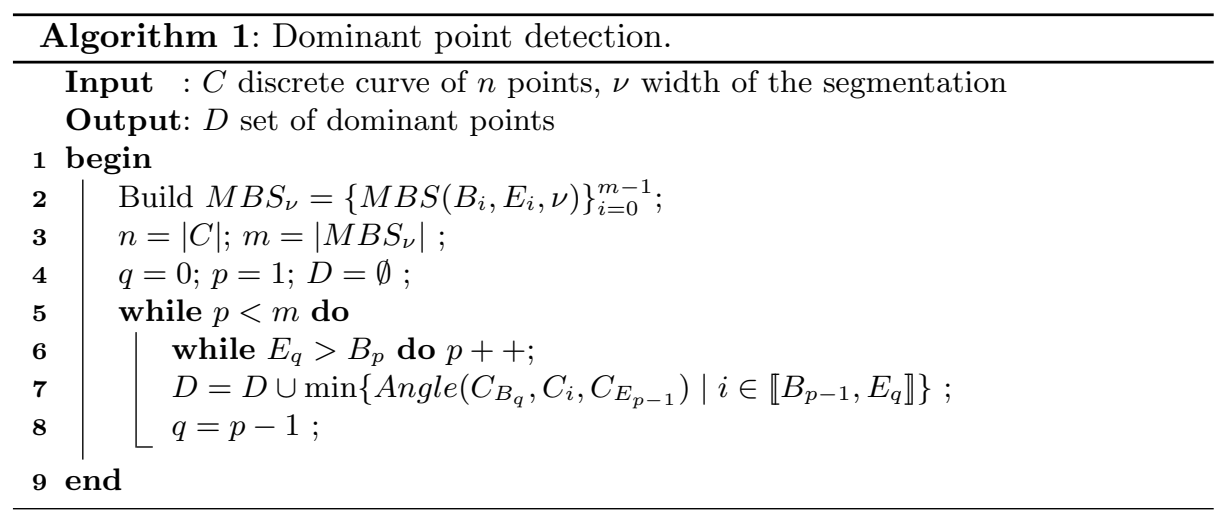

Particularly, in this algorithm, each common zone is computed by Line 6 , where $q$ is the index of the first maximal blurred segment involved in the common zone and $p-1$ is the index of the last. The points in the common zone are points $C_{i}$ for $i \in \llbracket B_{p-1}, E_{q} \rrbracket$.

Moreover, at Line 7, the function Angle calculates, for each point in the common zone, the angle between the point and its two extremities, then in a common zone, the dominant point is the point with local minimum angle. Afterwards, the next common zone is computed by considering the last segment 
(index $p-1$ ) of the previous zone as the first one (index $q$ ) of the next common zone. This process permits to detect the points of the curve through which a maximum number of maximal blurred segments is passing (see Fig. 3).

If the curve $C$ is not closed, the first and the last points of $C$ are dominant points and they must be added to $D$.

The algorithm is illustrated in Fig. 3 ; a curve $C$ is analysed by the algorithm, the sequence $M B S_{1.4}(C)$ is computed and 12 maximal blurred segments (named $s 0$ to $s 11$ in the figure) are obtained. Their localisation in the curve is indicated by one $\operatorname{star}(*)$ for each point belonging to the considered maximal blurred segment. Four common zones (dark pixels on the curve) are detected by the algorithm: $C_{14}$ to $C_{15}, C_{22}$ to $C_{26}, C_{49}$ to $C_{52}$ and $C_{59}$ to $C_{62}$. The pink boxes in the figure permit to count the number of maximal blurred segments passing through each point of common zones. The angles are calculated by considering the starting point of the first maximal blurred segment involved in the common zone and the end point of the last involved maximal blurred segment. The table 1 shows the angles at each point of common zones and permits to deduce the dominant points (minimal values are in bold in the table and the corresponding points are in red in Fig. 3). In the common zone 4, the selected dominant point is not located at the middle of the common zone.

\begin{tabular}{|c|c|c|}
\hline Common Zone & Points & Angle \\
\hline \hline Common Zone 1 & $C_{14}$ & 162.9 \\
\cline { 2 - 3 }$B_{S 0}$ and $E_{S 3}$ & $C_{15}$ & $\mathbf{1 5 9}$ \\
\hline \hline Common Zone 2 & $C_{22}$ & 155.6 \\
\cline { 2 - 3 }$B_{S 3}$ and $E_{S 5}$ & $C_{23}$ & 157.6 \\
\cline { 2 - 3 } & $C_{24}$ & $\mathbf{1 5 2}$ \\
\cline { 2 - 3 } & $C_{25}$ & 153.6 \\
\cline { 2 - 3 } & $C_{26}$ & 154.9 \\
\hline \hline \multirow{2}{*}{$\begin{array}{c}\text { Common Zone } 3 \\
B_{S 5} \text { and } E_{S 9}\end{array}$} & $C_{49}$ & 158.6 \\
\cline { 2 - 3 } & $C_{50}$ & 157.4 \\
\cline { 2 - 3 } & $C_{51}$ & 156 \\
\cline { 2 - 3 } & $C_{52}$ & $\mathbf{1 5 4 . 3}$ \\
\hline \hline Common Zone 4 & $C_{59}$ & 155.7 \\
\cline { 2 - 3 }$B_{S 9}$ and $E_{S 11}$ & $C_{60}$ & $\mathbf{1 5 0 . 5}$ \\
\cline { 2 - 3 } & $C_{61}$ & 155.3 \\
\cline { 2 - 3 } & $C_{62}$ & 160.2 \\
\hline
\end{tabular}

Table 1. Angles at each point of common zones (see Fig. 3).

The complexity of the proposed algorithm is the same as the one in [14], which depends on the complexity to decompose a curve into maximal blurred 
segments. We can use the technique proposed in [6] to obtain the tangential cover (corresponding to the sequence of maximal blurred segments for a given width) and the complexity of this method is in $O(n \log n)$.

\section{Evaluation results and applications}

We now present some experimental results of dominant point detection using the proposed method, and evaluate by widely used error criteria $[18,17]$ described in Sec 4.1. Using these criteria, in Sec 4.2 we show the improvement results of the modified algorithm regarding the original of Nguyen [14]. Afterwards, in Sec 4.3, we present an application in polygonal simplification with detected dominant points. The results are then compared with other popular methods in Sec 4.4.

\subsection{Evaluation criteria}

In order to assess the effectiveness of the proposed method, we consider the following five evaluation criteria:

1. Number of dominant points (nDP).

2. Compression ratio (CR) is defined as the ratio between number of curve points and number of detected dominant points. The larger $\mathrm{CR}$, the more effective data simplification.

$$
C R=\frac{n}{n D P}
$$

3. Integral sum of square errors (ISSE) is the sum of squared distances of the curve points from approximating polygon. The smaller ISSE, the better description of the shape by the approximating polygon.

$$
I S S E=\sum_{i=1}^{n} d_{i}^{2}
$$

where $d_{i}$ is distance from $i^{t h}$ curve point to approximating polygon.

4. Maximum error $\left(L_{\infty}\right)$ is the maximum distance of the curve points from approximating polygon. The smaller $L_{\infty}$, the better fitness of polygonal approximation.

$$
L_{\infty}=\max \left\{d_{i}\right\}_{i=1}^{n}
$$

5. Figure of merit (FOM) is estimated as ratio between CR and ISSE. FOM a compromise between the low approximation error and the benefit of high data reduction.

$$
F O M=\frac{C R}{I S S E}
$$




\subsection{Effectiveness compared to Nguyen's algorithm}

In this section, we present the experimental results of the modified algorithm (see Sec 3.2) and compare them with Nguyen's algorithm [14]. The experiments are carried out on the data with and without noise. For each input curve, a fixed width $\nu=1.5$ of maximal blurred segments is used for both algorithms, the results are shown in Fig. 4 and Table 2. It can be seen that selecting the middle point of common zone is not always a relevant strategy, in particular in the high-pass zones.

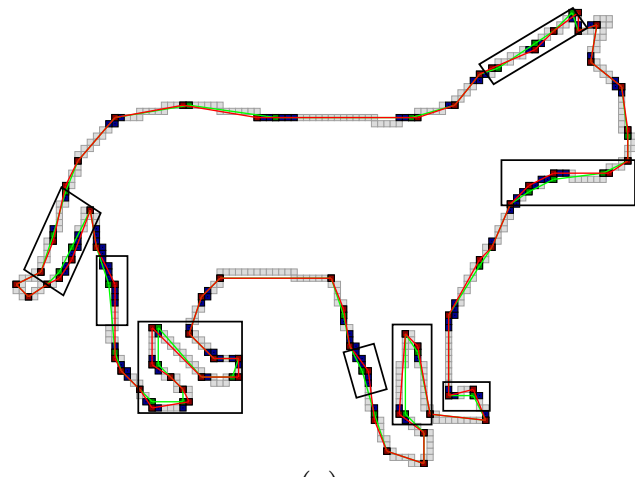

(a)

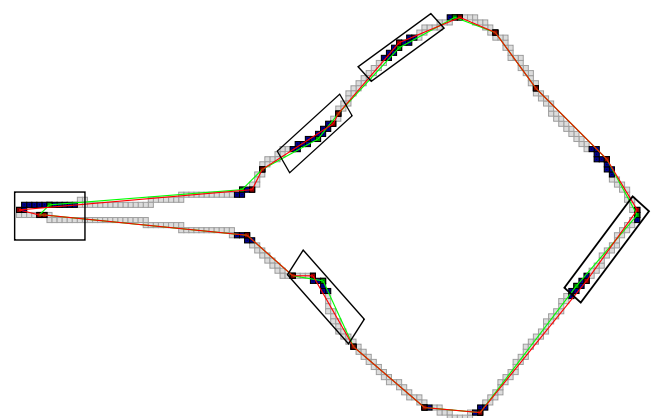

(c)

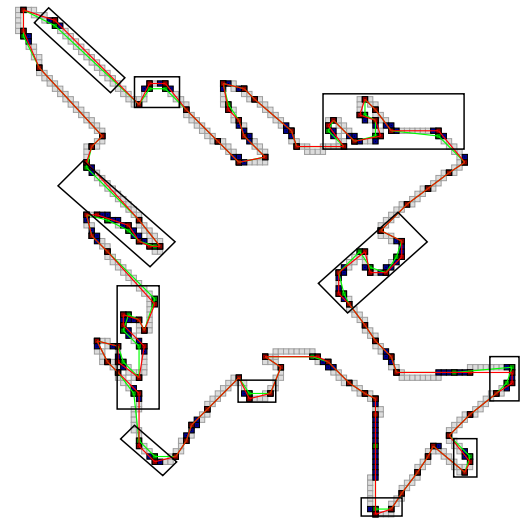

(b)

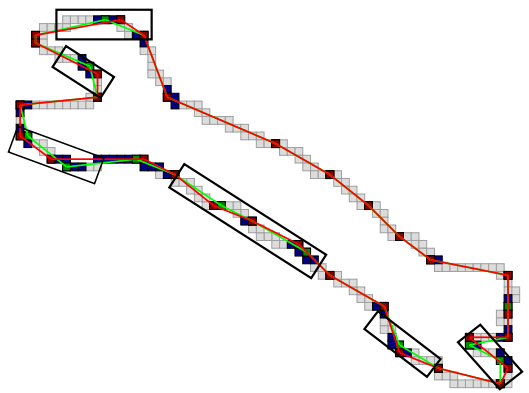

(d)

Fig. 4. Improved results with respect to Nguyen [14] (see also Table 2). The effectiveness of the modified algorithm is more significant in the highly derivative zones of the shape as highlighted by the black boxes. Green (resp. Red) points are dominant points detected by Nguyen's (resp. modified) algorithm. Lines in green (resp. red) denote the polygonal approximation from detected dominant points. Blue points are candidates of dominant points in the interest regions. 


\begin{tabular}{|c|l|c|c|c|c|c|}
\hline Curve & Method & nDP & CR & ISSE & $L_{\infty}$ & FOM \\
\hline \hline \multirow{2}{*}{$\begin{array}{c}\text { (a) } \\
n=536\end{array}$} & Nguyen & 68 & 7.882 & 149.15 & 1 & 0.0523 \\
\cline { 2 - 7 } & Ours & 68 & 7.882 & $\mathbf{1 2 5 . 7 6 3}$ & 1 & $\mathbf{0 . 0 6 2 7}$ \\
\hline \hline \multirow{2}{*}{$\begin{array}{c}\text { b) } \\
n=722\end{array}$} & Nguyen & 105 & 6.876 & 202.809 & 1 & 0.0339 \\
\cline { 2 - 7 } & Ours & 105 & 6.876 & $\mathbf{1 6 6 . 7 4}$ & 1 & $\mathbf{0 . 0 4 1 2}$ \\
\hline \hline \multirow{2}{*}{$\begin{array}{c}\text { (c) } \\
n=404\end{array}$} & Nguyen & 20 & 20.2 & 236.806 & 3.536 & 0.0853 \\
\cline { 2 - 7 } & Ours & 20 & 20.2 & $\mathbf{1 5 0 . 3 1 4}$ & $\mathbf{1 . 5 3 9}$ & $\mathbf{0 . 1 3 4 4}$ \\
\hline \hline \multirow{2}{*}{$\begin{array}{c}\text { d) } \\
n=252\end{array}$} & Nguyen & 43 & 5.86 & 68.896 & 1 & 0.0851 \\
\cline { 2 - 7 } & Ours & 43 & 5.86 & $\mathbf{5 7 . 5 8 2}$ & 1 & $\mathbf{0 . 1 0 1 8}$ \\
\hline
\end{tabular}

Table 2. Comparison with Nguyen's method [14] on Fig. 4 using significant measures described in Sec 4.1.

\subsection{Application in polygonal simplification}

The goal of finding the dominant points is to have an approximate description of the input curve, called polygonal simplification or approximating polygon. On the one hand, due to the nature of the maximal blurred segment sequence defined on a discrete curve, we observe that the common zones - contain candidates of dominant points - may be close to each others. On the other hand, using the algorithm proposed in Sec 3.2, the dominant point is selected according to its angle with the extremities of maximal segments. As a consequence, detected dominant points are sometimes redundant or stay very near, which is presumably undesirable in particular for polygonal simplification. More precisely, this leads to an overmuch dominant points and thus the polygon induced by such points is not an optimal solution of curve simplification. Therefore, we can eliminate certain dominant points to achieve a high compression of approximating polygon of the input curve. To this end, we associate to each detected dominant point a weight indicating/describing its importance with respect to the approximating polygon of the curve. Such a weight must: (1) be related to some error criteria of approximating polygon, and (2) not induce costly computation.

From Sec 4.1, the criterion ISSE describes the distortions caused by the approximated polygon of a curve. More precisely, ISSE allows to evaluate how much the approximated polygon is similar to the curve, thus smaller ISSE means better descriptive of the curve by the polygon. This error evaluation is suitable to our criterion of weight associated to dominant points since: (1) we would like to eliminate points that less affect the possible error (i.e. less ISSE), and (2) in particular the ISSE can be calculated locally by using their two neighbouring dominant points. Indeed, the value of ISSE between the approximated polygon and the curve before and after deleting a dominant point differs only at the part related to the point, in particular its two neighbours as illustrated in Fig. 5. The ISSE induced by the two neighbours of a dominant point thus characterizes the important of the point; the greater ISSE, the more important the point. 


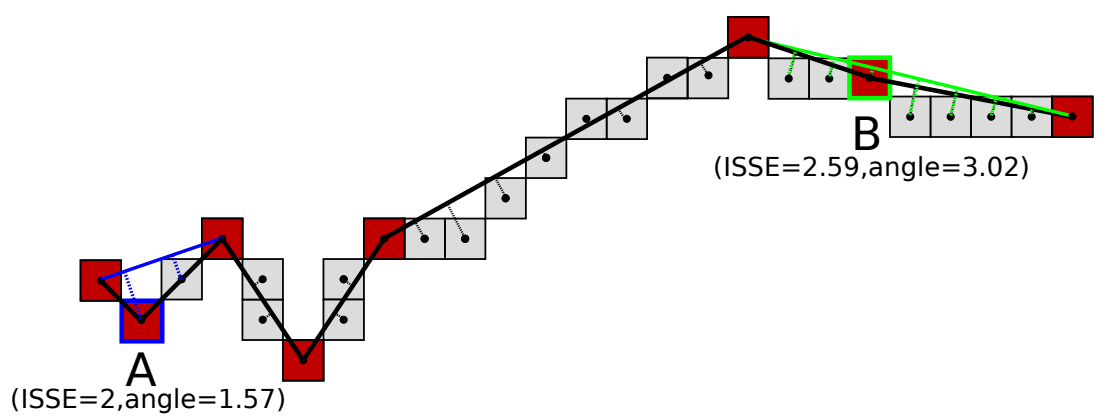

Fig. 5. Illustration of calculating the weights associated to detected dominant points. In grey (resp. red) are points (resp. dominant points) of the curves. The continuous black line connecting the dominant points denotes the approximated polygon, while dashed lines are perpendicular distances from points on the curve to the polygon, the sum of these distances defines the ISSE. Considering the point A in blue (resp. B in green) frame, deleting this point leads to a local modification in the approximated polygon, and thus ISSE induced by blue (resp. green) dashed lines. One remarks that ISSE of the point in blue frame is smaller than the green, however according to the neighbours - in angle - it is more important than the green one. Therefore, the weight associated to each dominant point involves both ISSE and angle criteria, in particular $\frac{I S S E}{\text { angle }}$. Then, the weight of $\mathrm{A}$ and $\mathrm{B}$ are 1.27 and 0.86 , respectively.

Still in Fig. 5, we remark that dominant point may have a small ISSE, however regarding its neighbours, in particular the angular relationship, it is more important than the others. In other words, this angle with the neighbours plays an important role in the decision of suppressing a dominant point. This leads to a consideration of weight associated to each dominant point detected that not only involves ISSE but also the angle to its two neighbours. In particular, this weight is determined by the ratio of ISSE and angle, i.e., ISSE/angle. Then, fixing a desired number $m$ of dominant points on the approximated polygon, roughly speaking, the process of polygonal simplification is performed by removing one by one the dominant points of small weight until reaching $m$.

In the next section, we test the proposed method and compare it to the other polygonal simplification techniques based on dominant points extraction.

\subsection{Comparison with other methods}

The experiments are first carried out on three benchmarks: chromosome, leaf and semicircle shown in Fig. 6, 7 and 8 respectively. Table 3 compares the proposed method with other popular algorithms such as: Masood [11], Marji $[9,10]$ and Teh [19], on the benchmarks by the evaluation criteria described in Sec 4.1. Overall the experiments, the proposed method presents an improvement more than of $38 \%$ on FOM, and has a better approximation error in ISSE and $L_{\infty}$.

Fig. 9 shows polygonal approximation results of the other proposed digital curves with noise. The results demonstrate the effectiveness and robustness of 
the proposed method on noisy data. Note that the experiments are performed by using Algorithm 1 (see Section 3.2 with a process of polygonal simplification as described in Sec 4.3).

\begin{tabular}{|c|c|c|c|c|c|c|}
\hline Curve & Method & $\mathrm{nDP}$ & $\mathrm{CR}$ & ISSE & $L_{\infty}$ & FOM \\
\hline \multirow{4}{*}{$\begin{array}{l}\text { Chromosome } \\
\qquad n=60\end{array}$} & Ours, $\nu=0.7$ & 14 & 4.286 & 5.116 & 0.8 & 0.838 \\
\hline & Masood [11] & 12 & 5 & 7.76 & 0.88 & 0.65 \\
\hline & Marji [9] & 12 & 5 & 8.03 & 0.895 & 0.623 \\
\hline & Teh [19] & 15 & 4 & 7.2 & 0.74 & 0.556 \\
\hline \multirow{4}{*}{$\begin{array}{c}\text { Leaf } \\
n=120\end{array}$} & Ours, $\nu=0.7$ & 23 & 5.218 & 9.065 & 0.671 & 0.576 \\
\hline & Masood [11] & 23 & 5.217 & 10.61 & 0.74 & 0.49 \\
\hline & Marji [10] & 22 & 5.45 & 13.21 & 0.78 & 0.413 \\
\hline & Teh [19] & 29 & 4.14 & 14.96 & 0.99 & 0.277 \\
\hline \multirow{4}{*}{$\begin{array}{c}\text { Semicircle } \\
n=102\end{array}$} & Ours, $\nu=0.7$ & 23 & 4.435 & 7.639 & 0.724 & 0.581 \\
\hline & Masood [11] & 22 & 4.64 & 8.61 & 0.72 & 0.54 \\
\hline & Marji [10] & 26 & 3.92 & 9.01 & 0.74 & 0.435 \\
\hline & Teh [19] & 22 & 4.64 & 20.61 & 1 & 0.225 \\
\hline
\end{tabular}

Table 3. Results of the proposed method and of the other methods.

\section{Conclusion and future work}

We present in this paper a dominant point detection algorithm, improvement of a previous algorithm which uses an heuristic strategy. This algorithm relies on the structure of the discrete curve and the width, parameter of the algorithm, permits to work on data with or without noise. For a given width, our method associates to each detected dominant point a weight which permits to evaluate the importance of the dominant point in the structure of the studied curve. We then deduce a method to reduce the number of dominant points and to obtain the smallest possible error according to a given number of dominant points.

The proposed method opens numerous perspective of future work, for example the study of the method behaviour in a multiscale approach to detect the more appropriate width for dominant point selection.

\section{References}

1. Aguilera-Aguilera, E.J., Poyato, Á.C., Madrid-Cuevas, F.J., Carnicer, R.M.: The computation of polygonal approximations for $2 \mathrm{~d}$ contours based on a concavity tree. J. Visual Communication and Image Representation 25(8), 1905-1917 (2014)

2. Attneave, E.: Some informational aspects of visual perception. Psychol. Rev. 61(3), 183-193 (1954) 


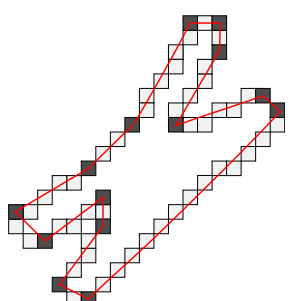

(a) Ours, $\nu=0.7$

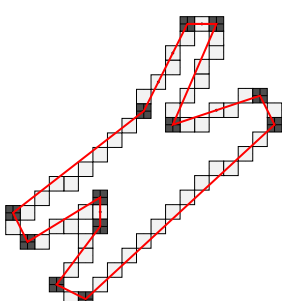

(b) $\operatorname{Masood}[11]$

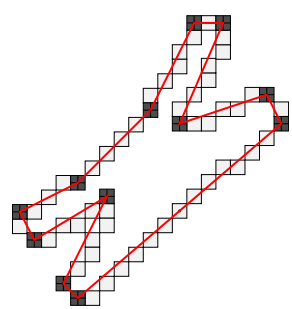

(c) Marji [9]

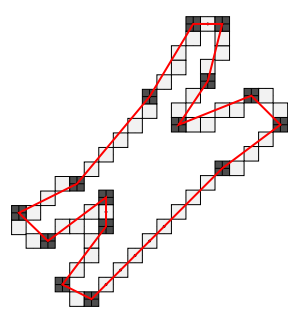

(d) $\operatorname{Teh}[19]$

Fig. 6. Dominant points of the chromosome curve.

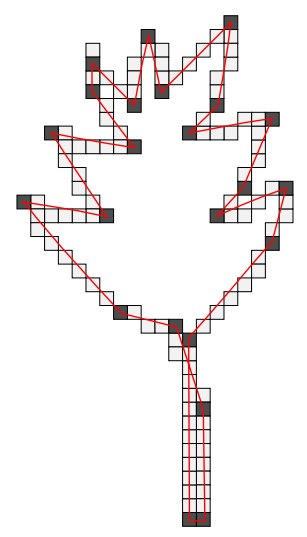

(a) Ours, $\nu=0.7$

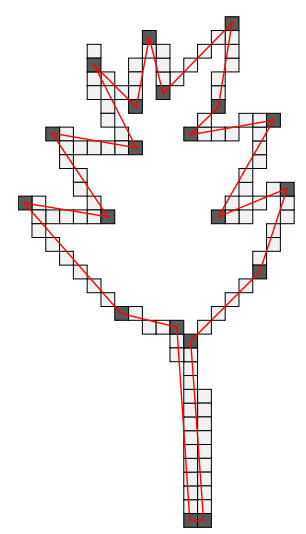

(b) $\operatorname{Masood}[11]$

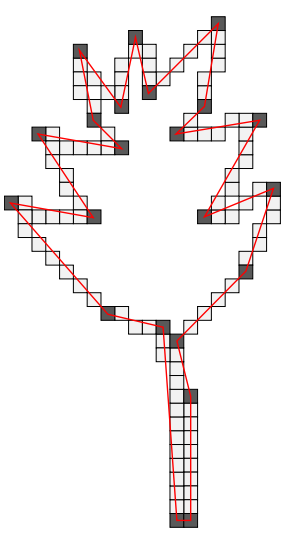

(c) Marji [10]

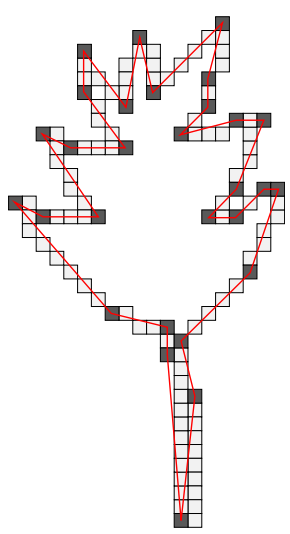

(d) Teh[19]

Fig. 7. Dominant points of the leaf curve.

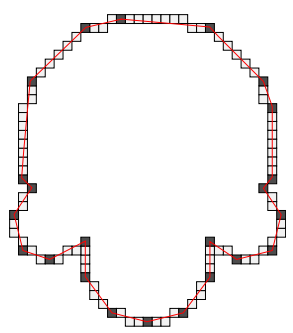

(a) Ours, $\nu=0.7$

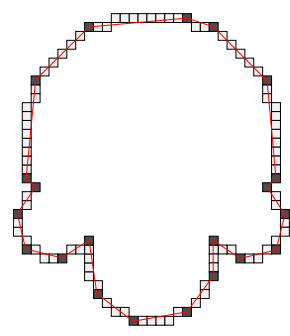

(b) Masood[11]

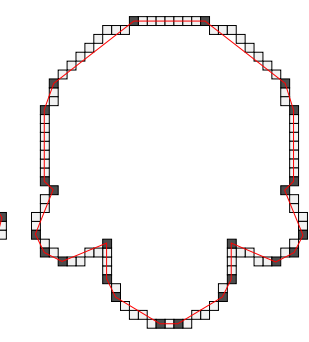

(c) Marji [10]

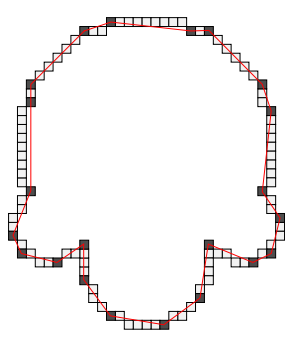

(d) Teh[19]

Fig. 8. Dominant points of the semicircle curve. 

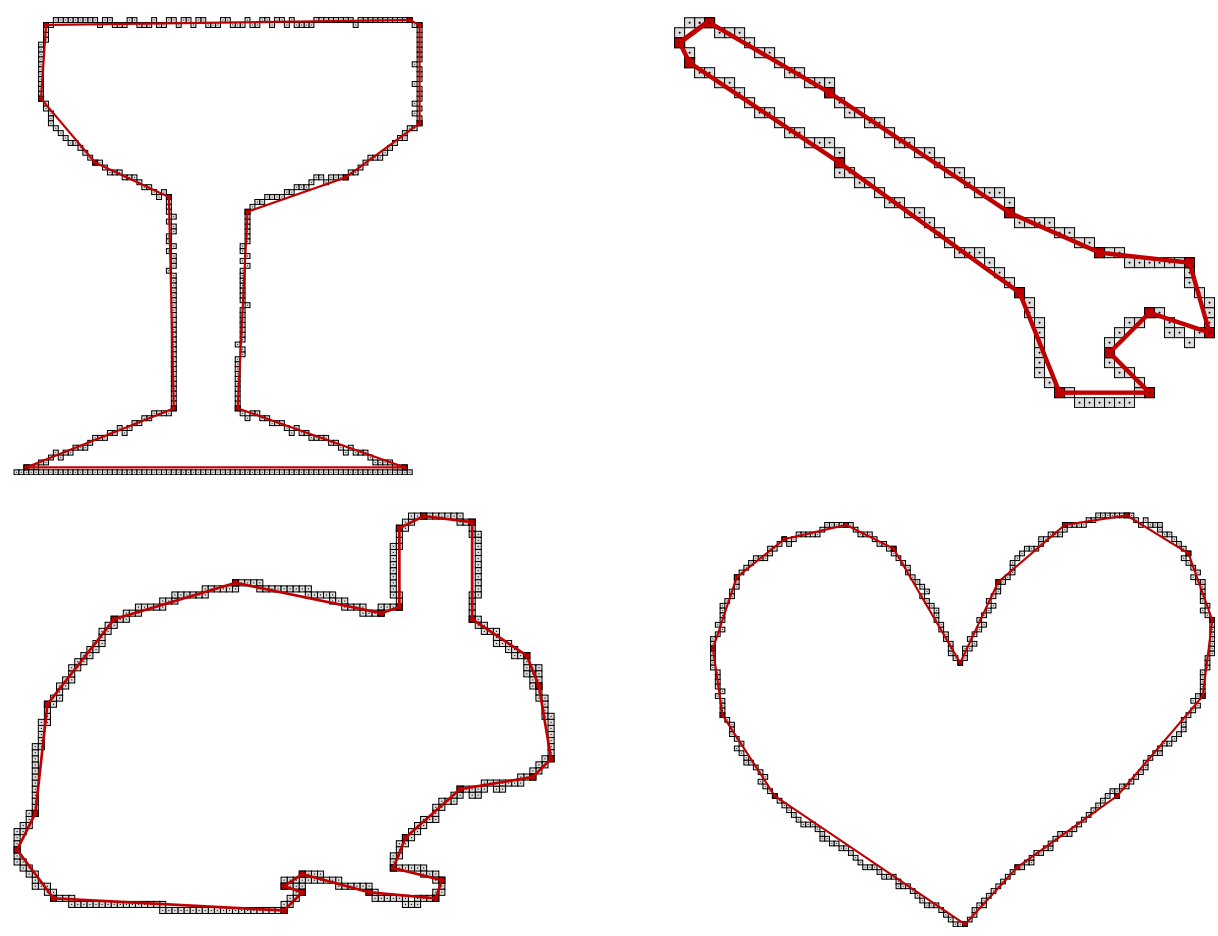

Fig. 9. Polygonal simplification results (in red) on noisy data using width parameter $\nu=1.5$, and a reduction of $10 \%$ of dominant points detected by Algo 1 .

3. Backes, A.R., Bruno, O.M.: Polygonal approximation of digital planar curves through vertex betweenness. Inf. Sci. 222, 795-804 (2013)

4. Bhowmick, P., Bhattacharya, B.B.: Fast polygonal approximation of digital curves using relaxed straightness properties. IEEE Trans. Pattern Anal. Mach. Intell. 29(9), 1590-1602 (2007)

5. Debled-Rennesson, I., Feschet, F., Rouyer-Degli, J.: Optimal blurred segments decomposition of noisy shapes in linear time. Computers \& Graphics 30(1), 30-36 (2006)

6. Faure, A., Buzer, L., Feschet, F.: Tangential cover for thick digital curves. Pattern Recognition 42(10), 2279-2287 (2009)

7. Feschet, F., Tougne, L.: Optimal time computation of the tangent of a discrete curve: Application to the curvature. In: DGCI. LNCS, vol. 1568, pp. 31-40 (1999)

8. Feschet, F.: Fast guaranteed polygonal approximations of closed digital curves. In: SCIA. LNCS, vol. 3540, pp. 910-919 (2005)

9. Marji, M., Siy, P.: A new algorithm for dominant points detection and polygonization of digital curves. Pattern Recognition 36(10), 2239-2251 (2003)

10. Marji, M., Siy, P.: Polygonal representation of digital planar curves through dominant point detection - a nonparametric algorithm. Pattern Recognition 37(11), $2113-2130(2004)$

11. Masood, A.: Dominant point detection by reverse polygonization of digital curves. Image Vision Comput. 26(5), 702-715 (2008) 
12. Masood, A.: Optimized polygonal approximation by dominant point deletion. Pattern Recogn. 41(1), 227-239 (2008)

13. Nguyen, T.P., Debled-Rennesson, I.: Curvature estimation in noisy curves. In: CAIP. LNCS, vol. 4673, pp. 474-481 (2007)

14. Nguyen, T.P., Debled-Rennesson, I.: A discrete geometry approach for dominant point detection. Pattern Recognition 44(1), 32-44 (2011)

15. Reveillès, J.P.: Géométrie discrète, calculs en nombre entiers et algorithmique (1991), thèse d'état. Université Louis Pasteur, Strasbourg

16. Rosenfeld, A., Johnston., E.: Angle detection on digital curves. IEEE Transactions on Computers pp. 875-878 (1973)

17. Rosin, P.L.: Techniques for assessing polygonal approximations of curves. IEEE Trans. Pattern Anal. Mach. Intell. 19(6), 659-666 (1997)

18. Sarkar, D.: A simple algorithm for detection of significant vertices for polygonal approximation of chain-coded curves. Pattern Recognition Letters 14(12), 959-964 (1993)

19. Teh, C., Chin, R.: On the detection of dominant points on the digital curves. IEEE Trans. Pattern Anal. Mach. Intell. 2, 859-872 (1989)

20. Wang, B., Brown, D., Zhang, X., Li, H., Gao, Y., Cao, J.: Polygonal approximation using integer particle swarm optimization. Inf. Sci. 278, 311-326 (2014) 